\title{
The Control of Mechanical Impedance Using an Electrodynamic Actuator
}

\author{
Comeaga Constantin Daniel, Mitiu Mihaela, and Dontu Grigore Octavian
}

\begin{abstract}
The paper presents a study regarding the practical use of electrodynamic actuators for controlling the mechanical impedance and mobility of a 1 degree-of-fredom system, with application to transmissibility control. The electrodynamic actuators included in the structure of mechanical systems could be used as a semi-active solution to control the global mechanical parameters but with limitations due to the intrinsic electric properties of the actuator. An improvement of this technique is the the active solution based on different control laws and different variables (speed, acceleration, current), able to provide equivalent electrical parameters difficult to obtain by construction or even to introduce new properties in the system. The current study presents the principles used for this type of control and also shows some limitations due to the lack of stability for some ranges and types of feedback.
\end{abstract}

Index Terms-Active vibration control, semi-active vibration control, electrodynamic actuator.

\section{INTRODUCTION}

Vibration control of machines or mechanical structures is a very important process.

There are three main ways in which better vibration isolation effect can achieve, namely [1]:

- reducing the number of vibration generating sources;

- intervention on the routes of transmission of vibrations by the introduction of isolators or isolating means;

- the change of system parameters on which vibrations (receiver system) or radiating elements (which transmit and amplify vibrations perceived) acts.

In all three cases means and ways for passive control can be applied, with best results only in special cases or for limited areas of vibration frequencies [2]. Active or semi-active control systems are used in parallel or entirely replace the passive systems in reducing the effects of vibration.

Most of the non-pasive vibration control applications deal with active control, using different types of actuation [3]. The active vibration control involves measuring a vibration parameter and applying a force computed using a control law (feedback or feed-forward) usually based on the sensor signal.

The system allows compensation of significant vibration

Manuscript received March 10, 2015; revised October 13, 2015. This work was supported in part by the National Agency for Research UEFISCDI under Grant 249/2014.

C. D. Comeaga and O. G. Dontu are with the University Politehnica of Bucharest, Research and Development Center for Mechatronics, Bucharest, RO 77206 Romania (e-mail: comeaga@hotmail.com, octavdontu@yahoo.com).

M. Mitiu is with the National Institute for Research and Development for Environmental Protection, Bucharest, RO 060031 Romania (e-mail: mihaela.mitiu@yahoo.com). levels but requires a powerful energy supply and has sometimes an unstable behavior if the system parameters are changing in functioning. Different types of sensors and actuators were proposed, measuring/actuating in a point (concentrated control) or on a line/surface (distributed control). A great interest got in the last decades the active materials, mainly piezoelectric ones, included in composite structures [4] (usually laminated but also as particles and wires). It is the so called distributed control using active structures. The main advantage of using piezoelectric materials is the possibility to use some piezoelectric patches as sensors and others as actuators. Some techniques were developed to use the same structure for actuation and also as sensor, the self-sensing actuating solution.

The studies for semi-active vibration control are less numerous and deal mainly with automotive applications [5]. The semi-active control involves usually the tuning of a parameter from a passive damping system. As tuning of stiffness is more difficult, the damping is usually controlled. Most of the published research deals with adaptive fluids as electrorheological and magnetorheological ones, easily to integrate into actual dampers. Also piezoelectric materials can be use for semi-active vibration control, the material having the role of energy converter, from mechanical (deformation) energy to electrical one and then dissipation using and external electric circuit. The piezoelectric material has low efficiency of conversion out of resonance frequencies., usually at very high values.

Some chapters about semi-active control can be found in the books about vibration control [3], [4] but the space is usually very limited comparing to active control.

\section{The Use of Electrodynamic Actuator for Active AND SEMI-ACTIVE VIBRATION CONTROL}

Among the current types of actuators involved in the active and semi-active vibration control, the electrodynamic actuator is the one able to provide high forces, high stokes and also working on a broad range of frequencies and having an affordable price. It is difficult to integrate it into an existing system due to the volume and weight of magnets and coil. Also the control requires high electric currents, producing high energy dissipation and thermal problems, a disadvantage comparing with the piezoelectric systems, operated at very high voltages - easy to control with actual electrical circuit components - but very low currents. The electrodynamic actuator, sometimes called voice-coil actuator due to large presence in loudspeakers, is used for anti-vibration tables [6], [7] to control the transmissibility of vibration, or for tuning the impedance and mobility of vibration isolators [8]. These applications are mainly based 
on active vibration control, with different technical solutions and feedback laws.

Another possibility to use the voice-coil actuator for vibration control is to integrate in inertial dampers, as in the applications for aircraft industry [9], connected to the main system and having the role of absorbing and dissipating the vibration energy from the main system. The passive solution has efficiency in a narrow frequency band, tuned at the main resonance of the parent system. The active systems allow not only to dissipate the energy of the main system but also to actuate it in order to reduce the local vibration.

Different feedback laws were proposed in the previous studies dealing with the use of electrodynamic actuators for active control, from typical closed loop control using an external vibration sensor to self-sensing solutions. The last involves sensing the vibration (the mobile mass velocity) using the back-EMF voltage (the induced voltage due to the coil movement is proportional with the coil velocity). The voltage can be extracted using a current feedback or using a Wheatstone bridge. The first technique has limitations due to the intrinsic electrical parameters of coil (resistance and inductance) but the last one allows removal of this parameters and a pure velocity feedback. In the last one the feeding back of the voltage produces similar results as velocity feedback resulting in active damping. Practical implementation is not so easy due to some instability produced by the non-ideal integrators for accelerometers and differentiators for displacement sensors.

Electrodynamic self-sensing was used for active vibration damping of hard-disk drives [10], high-speed elevators [11] and on a large scale in improving the performances of electrodynamic loudspeakers at low frequencies.

Two possibilities for estimation of the coil velocity by measuring the induced voltage using a Wheatstone bridge were used: a pure resistive bridge, allowing control of the equivalent coil resistance, and negleting the coil inductance effect [12], [13], and a complete bridge with capacitors and resistors for controlling the resistance but also inductance [14], [15]. The first solution is simpler but feasible at low frequencies while the second controls of the behavior at high frequencies. When not compensating for the coil inductance too, large vibration amplification was reported at higher frequencies [16].

Other technique involves use of a negative output impedance amplifier using different solutions [17]. Studies were mainly concentrated for reduction of effective loudspeaker resistance by direct positive current feedback or using combined self-sensing schemes, because an amplification of the coil velocity at very high frequency is not critical for a loudspeaker, as it is for vibration control.

Nowadays were proposed more complex solution for complete inductance compensation [18] for active control, used to improve the response of loudspeakers [19].

The techniques developed for improving loudspeaker behavior could be used also for vibration control applications, both active and semi-active.

The characteristics of the main techniques, some borrowed from electro-acoustics, used for active vibration control [20] can be summarized as:

- absolute velocity feedback using an accelerometer placed on the mobile mass; negative feedback add damping until the equivalent resistor value is closed to zero. At very low resistor values a supplementary resonance is produced, due to the effect of the coil inductance, which influences the total equivalent stiffness. Higher negative damping produces instability. Positive feedback leads to negative damping and possible instability. The accelerometer introduces due to its own impedance and natural frequency a possible instability at very high frequencies.

- current feedback using a resistor connected in series with the coil to measure the current as a voltage drop on this resistor (the electrodynamic actuator is considered as a self-sensing actuator). Apparently, proportional current feedback should be similar to absolute velocity feedback since the back-EMF is proportional with the absolute velocity. Current feedback should show the effect of absolute velocity feedback without the need for an additional sensor. Practical studies showed the limitations: the force acting on the mobile mass is proportional with a low pass filtered velocity signal, with the cut-off frequency of the filter inverse proportional with the feedback gain. So, high damping restricts the useful frequency domain to low frequencies. A high frequency band can be obtained only with the price of a low feedback gain - a trade off between gain and cut-off frequency of the feedback appears! The problems linked with the appearance of a new resonance if the negative feedback is smaller but much closed to the coil resistance, mentioned for the velocity feedback, remains true.

- pure velocity feedback based on current measurement using a Wheatstone bridge, in two configurations: a resistor bridge and a capacitive-resistive bridge. The previous studies showed that an increased bridge voltage feedback gain leads to an increased velocity feedback, producing an increased damping, but without the disadvantage of the useful frequency band reduction, as in the previous case.

Majority of studies regarding the use of electro-dynamic actuators dealt with impedance control of mechanical systems and less with the transmissibility control.

The voice-coil (electrodynamic actuator) can be used also with success for a kind of semi-active vibration control. The control is not a typical semi-active solution because is not controlling a mechanical parameter directly (as in dampers with electrorheological and magnetorheological fluids). The actuator is used more as an energy transformer from mechanical to electrical energy. Then the energy is applied on different circuits, producing a behavior similar with the control of mechanical parameters (mass/stiffness or damping). These actuators proved to be a good solution for semi-active vibration control but with limitations due to the intrinsic electrical parameters of the coil. The use of the aforementioned solutions for removing totally or partially the coil electrical components could be seen as a possibility to overcome these limitations, the focus being not on the global feedback parameters (even a kind of active control is applied at the electrical circuit for producing a negative resistance or inductance) but on the solutions to control better the semi-passive parameters.

In the same time, by using electrodynamic actuators, it is possible to improve the performances of classic solution of impedance and transmissibility control based on mechanical 
dynamic absorbers. Previous studies shown two possibilities of improvement: to insert an electrodynamic actuator havin purpose to transform the mechanical energy to electrical one into the structure of the electrodynamic absorber and adjust the electrical impedance connected [21] or, the second option, to completely replace the dynamic absorber with an electrodynamic actuator and an electrical impedance chosen to obtain similar effect as the absorber [22].

Previous studies regarding the use of electro-dynamic actuator for control of transmissibility [23] confirmed both by experimental results and those obtained from simulation, the reliability of mechanical transmissibility control by using a bidirectional electro-mechanical energy converter and external electrical impedance. Any kind of electrical component (resistor, capacitor, inductor) in the electrical circuit of the actuator is changing the response at resonance but also the resonance frequency.

The user needs to control two electric components to change only one mechanical parameter at resonance. Adding a resistor is decreasing the response at resonance, increasing the damping inverse proportional with the resistor value, but also is decreasing the resonance frequency.

Adding an inductance is rising the resonance frequency but also the response at resonance.

Adding a capacitor is decreasing the resonance frequency and is raising the response at resonance.

A study for identifying the optimum values for the external impedance components is presented in [24] and indicated that when the external electrical circuit resonance or the electromechanical resonance is dominant, the behavior of the system is worsening, a situation which usually occurs at low values of total electrical resistance $R$.

In the same time, the use of high values for the electrical resistance $\mathrm{R}$ cancels the effect of the electric circuit on the mechanical system. There is an optimum range of variation for the value of the resistance $\mathrm{R}$. It must be satisfied the condition that the purely electric or electromechanical resonance occur at a frequency much different that of the pure mechanical system.

For a mechanical system, with variable parameters during operation, is necessary therefore the adjustment of both inductance and capacitance parameters (in order to fulfill the condition of difference between frequency resonance) but also the variation of the electrical resistance $\mathrm{R}$, in order to fit within the flat response curve.

Also the optimum can be obtained only for very small values of the electrical parameters, difficult to obtain in practice (low resistance or inductance) using only the design of the actuator (mainly the coil).

From these studies aroused the idea to combine the circuits used in high performance loudspeakers control circuits (negative amplification - negative resistance circuits and negative inductance circuits) with the semi-active systems, more stable and cheaper than active ones, to improve their efficiency by reducing the coil electrical parameters.

\section{MATHEMATICAL MODEL OF THE STUDIED SySTEM}

The studied system is a simple mechanical system (Fig. 1), with one degree of freedom, for isolation from external influences of a body of mass $\mathrm{m}$.
The electrodynamic actuator is the active element for the control of system characteristics or it can be considered as a semi-active part of the same system when it is used as a converter of mechanical energy into electrical energy that can be dissipated later by an external impedance $(Z e)$.

The main parameters of the components are the following: $k_{m}-$ [N/A ] - the electromechanical factor; $k_{e}-[\mathrm{V} / \mathrm{m} / \mathrm{s}]-$ the mechanical -electrical factor; $c-[\mathrm{m} / \mathrm{N}]-$ the mechanical compliance; $i$ - [A] - the control current applied to the coil; $\mathrm{k}$ - [N/m] - the elastic constant of spring; $m-[\mathrm{kg}]$ - the mass of the stabilized system; $R b-[\Omega]-$ the coil resistance; $U-$ [V] - the control voltage of the coil; Uem - [V] - the electromotive force voltage induced in the coil; $v_{0}-[\mathrm{m} / \mathrm{s}]-$ the speed of movement of the mass $m$ relative to the base; $Z e$ $-[\Omega]$ - the external impedance.

To determine the equation describing the operation of the electrodynamic actuator control the scheme presented in Fig. 1 is used.

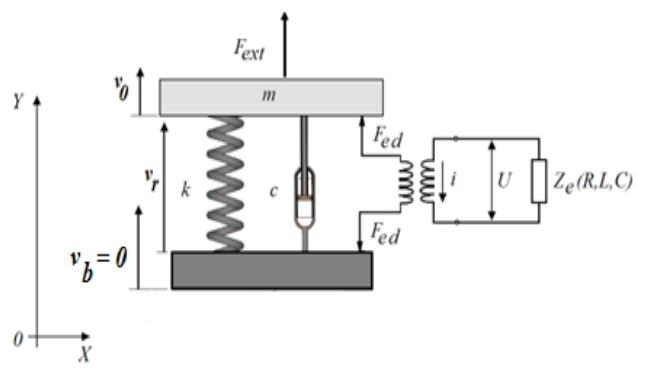

Fig. 1. The mechanical system with an electrodynamic actuator and electrical impedance.

The mechanical equilibrium equation of the system and the electric circuit equation are:

$$
\begin{aligned}
& m \cdot \frac{d v_{0}}{d t}=F_{e x t}+k_{m} \cdot i-c \cdot V_{r}-k \cdot X_{r} \\
& k_{e} \cdot v_{0}+L_{b} \cdot \frac{d i}{d t}+\left(R_{b}+Z_{e x t}\right) \cdot i=U \cdot
\end{aligned}
$$

The mechanical and electrical-mechanical impedances of the system are:

$$
\begin{aligned}
& Z_{m}=m \cdot s+\frac{k}{s}+c \\
& Z_{e}=R+L \cdot s+Z_{e x t} .
\end{aligned}
$$

From equations system (1), using relatively simple operations, results the relations for the calculation of current and velocity:

$$
\begin{aligned}
& i=\frac{U}{Z_{e} \cdot\left(1+k_{m} \cdot \frac{k_{e}}{Z_{e}} \cdot Z_{m}\right)}- \\
& -F_{e x t} \cdot \frac{k_{e}}{Z_{m} \cdot Z_{e} \cdot\left(1+k_{m} \cdot \frac{k_{e}}{Z_{e} \cdot Z_{m}}\right)} \\
& v_{o}=\frac{U \cdot k_{m}}{Z_{m} \cdot Z_{e} \cdot\left(1+k_{m} \cdot \frac{k_{e}}{Z_{e} \cdot Z_{m}}\right)}+ \\
& +F_{e x t} \cdot \frac{1}{Z_{m} \cdot\left(1+k_{m} \cdot \frac{k_{e}}{Z_{e} \cdot Z_{m}}\right)}
\end{aligned}
$$


The previous study has indicated the possibility of impedance and mobility control in a way similar to a changing of the mechanical parameters $(m, k$ and $c$ ) but modifying only the electrical parameters of the total electric impedance applied at the electrodynamic actuator terminal, the study showing also significant limitations due to the electric components introduced by the actuator coil (resistance and inductance).

Further are presented a few possibilities for control of the electrical impedance through feedback.

1) Case of feedback law based on mass movement $U=H(s) \cdot v_{o}$

The mechanical equation of mass movement became:

$$
\begin{gathered}
v_{0} \cdot Z_{m}^{*}=F_{e x t} ; \\
Z_{m}^{*}=Z_{m}+\frac{k_{m}}{Z_{e}} \cdot\left(k_{e}-H(s)\right) .
\end{gathered}
$$

where $Z m^{*}$ is the equivalent mechanical impedance.

Equation (4) indicate the possibility to control $Z_{m} *$ using feedback law.

If the system includes an accelerometer placed on the mobile mass, it is easy to implement a proportional acceleration feedback and also a proportional velocity feedback.

a) The case of a proportional velocity feedback

$$
H(s)=c t .=\alpha
$$

The equivalent mechanical impedance is:

$$
Z_{m}^{*}=Z_{m}+\frac{k_{m}}{Z_{e}} \cdot\left(k_{e}-\alpha\right)=m^{*} \cdot s+\frac{k^{*}}{s}+c^{*} \cdot s
$$

Few particular values of electrical impedance $\mathrm{Ze}$ are interesting:

If $Z_{e} \approx R$ then

$$
c^{*}=c+\frac{k_{m}}{R} \cdot\left(k_{e}-\alpha\right)
$$

and shows the possibility to adjust the equivalent damping factor $\mathrm{c}^{*}$. The practical implementation requests null external impedance and also strong reduction/canceling of the coil electrical inductance, difficult to obtain.

If $Z_{e} \approx L \cdot s$ then

$$
k^{*}=k+\frac{k_{m}}{R} \cdot\left(k_{e}-\alpha\right)
$$

and shows the possibility to adjust the equivalent stiffness factor $\mathrm{k}^{*}$. The practical implementation requests null external impedance and also strong reduction/canceling of the coil electrical resistance, also difficult to obtain.

$$
\begin{aligned}
& \text { If } Z_{e} \approx \frac{1}{s \cdot C} \text { then } \\
& \begin{array}{l}
Z_{m}^{*}=Z_{m}+s \cdot C \cdot k_{m} \cdot\left(k_{e}-\alpha\right) \\
m^{*}=m+C \cdot k_{m} \cdot\left(k_{e}-\alpha\right)
\end{array}
\end{aligned}
$$

and shows the possibility to adjust the equivalent mass $\mathrm{m}^{*}$. The practical implementation requests capacitive external impedance and also strong reduction/canceling of the coil electrical resistance and impedance.

b) The case of a proportional acceleration feedback $H(s)=\alpha \cdot s$

The equivalent mechanical impedance is:

$$
Z_{m}^{*}=Z_{m}+\frac{k_{m}}{Z_{e}} \cdot\left(k_{e}-\alpha \cdot s\right)
$$

Few particular values of electrical impedance Ze are interesting:

If $Z_{e} \approx R$ then

$$
Z_{m}^{*}=Z_{m}+\frac{k_{m} \cdot k_{e}}{R}-\frac{k_{m}}{R} \cdot \alpha \cdot s
$$

and shows a modification of the equivalent damping factor $c^{*}\left(c^{*}=c+\frac{k_{m} \cdot k_{e}}{R}\right)$ but also the possibility to adjust the equivalent mass $\mathrm{m}^{*}\left(\mathrm{~m}^{*}=\mathrm{m}-\frac{\mathrm{k}_{\mathrm{m}}}{\mathrm{R}} \cdot \alpha\right)$. It is necessary to have a null external electrical impedance and also strong reduction/canceling of the coil electrical inductance;

If $Z_{e} \approx L \cdot S$ then

$$
Z_{m}^{*}=Z_{m}+\frac{k_{m} \cdot k_{e}}{L \cdot s}-\frac{k_{m}}{L} \cdot \alpha
$$

and shows a modification of the equivalent stiffness factor $\mathrm{k}^{*}$, but also the possibility to adjust the equivalent damping factor $\mathrm{c}^{*}$ :

$$
k^{*}=k+\frac{k_{m} \cdot k_{e}}{L} ; c^{*}=c-\frac{k_{m}}{L} \cdot \alpha
$$

It is necessary to have a null external electrical impedance and also strong reduction/canceling of the coil electrical resistance.

Case 1) could offer favorable results, mainly the a) situation, if the user has a possibility to have a controlled external electrical impedance (easily to implement in practice) but in the same time a possibility to cancel/reduce very much the inductance or resistance of the actuator coil.

2) Case of feedback law based on current in coil $U(s)=H(s) \cdot i$

The equation of the system equivalent mechanical impedance become:

$$
Z_{m}^{*}=Z_{m}-\frac{k_{m} \cdot k_{e}}{H(s)-Z_{e}}
$$

Few situations are interesting:

if $H(s)=\alpha \cdot i$ then

$$
Z_{e}-H(s)=(R-\alpha) \cdot i+L \cdot s \cdot i+Z_{e x t} \cdot i
$$

if $H(s)=\alpha \cdot i \cdot s$ then

$$
Z_{e}-H(s)=R \cdot i+(L-\alpha) \cdot s \cdot i+Z_{e x t}
$$

if $H(s)=-\frac{1}{\alpha \cdot s} \cdot i$ then 


$$
Z_{e}-H(s)=R \cdot i+L \cdot s \cdot i+\frac{1}{\alpha \cdot s^{2}}+Z_{e x t}
$$

The last equation shows that a current feedback acts as a control of the electrical impedance, hence becoming possible to adjust the total resistance or inductance of the circuit including the theoretical possibility to cancel completely the resistance or/and inductance of the coil. This allows the implementation of the situation indicated in case a.1), but requesting a combined feedback with a current loop for canceling the disturbing components and a velocity/acceleration feedback for controlling the equivalent mechanical parameters. In Fig. 2 it is shown the simulation of the ratio acceleration/force, for different values of the total electrical resistance, obtained using a proportional current feedback for control of the resistance. The circuit contains an inductance which can be controlled via an additional current circuit with derivative component. A negative value of resistance produces a worst situation, with a very low damping (a strong oscillation was observed near the mechanical resonance). A value close to null of the resistance conducts to a new value of the resonance frequency due the effect of inductance (significant increase of the stiffness as the inductance is positive and very small).

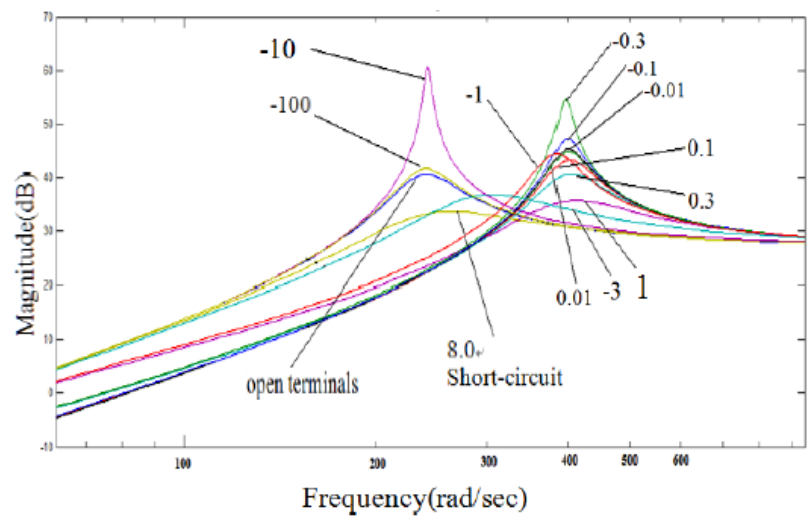

Fig. 2. The effect of electrical resistance $\mathrm{Rb}$ (values indicated in graph are in $\Omega)$, obtained with different current feedback, on the function acceleration/force, for: $\mathrm{m}=0.0434 \mathrm{~kg}$; $\mathrm{k}=2452.5$; $\mathrm{c}=2.2305$; $\mathrm{km}=5$; $\mathrm{ke}=5$; $\mathrm{Lb}=0.0056 \mathrm{H} ; \mathrm{C}=0 \mathrm{~F}$.

\section{TECHNICAL IMPLEMENTATION AND EXPERIMENTS}

For implementing with simplicity the discussed feedback laws an analogue electrical circuit was designed, presented in Fig. 3.

The electrical circuit was also used for experimentally checking of the principles described above. The resistor R40 is connected in the coil circuit. The voltage drop on R40, proportional with the current is fed through the upper (amplification with an adjustable value, inversion or not) and middle (derivation, amplification with an adjustable value, inversion or not) path. The upper path allows control of the circuit global resistance and the middle path allows control of the circuit global inductance. The lower path allows connection of an external signal (for example the signal from the accelerometer with or without integration). Finally, the signals could be connected using switches to a summing circuit. The resulting signal is amplified and applied at the actuator circuit.
To perform the experimental study has chosen a system that integrates all the items and properties listed, an electrodynamic speaker.

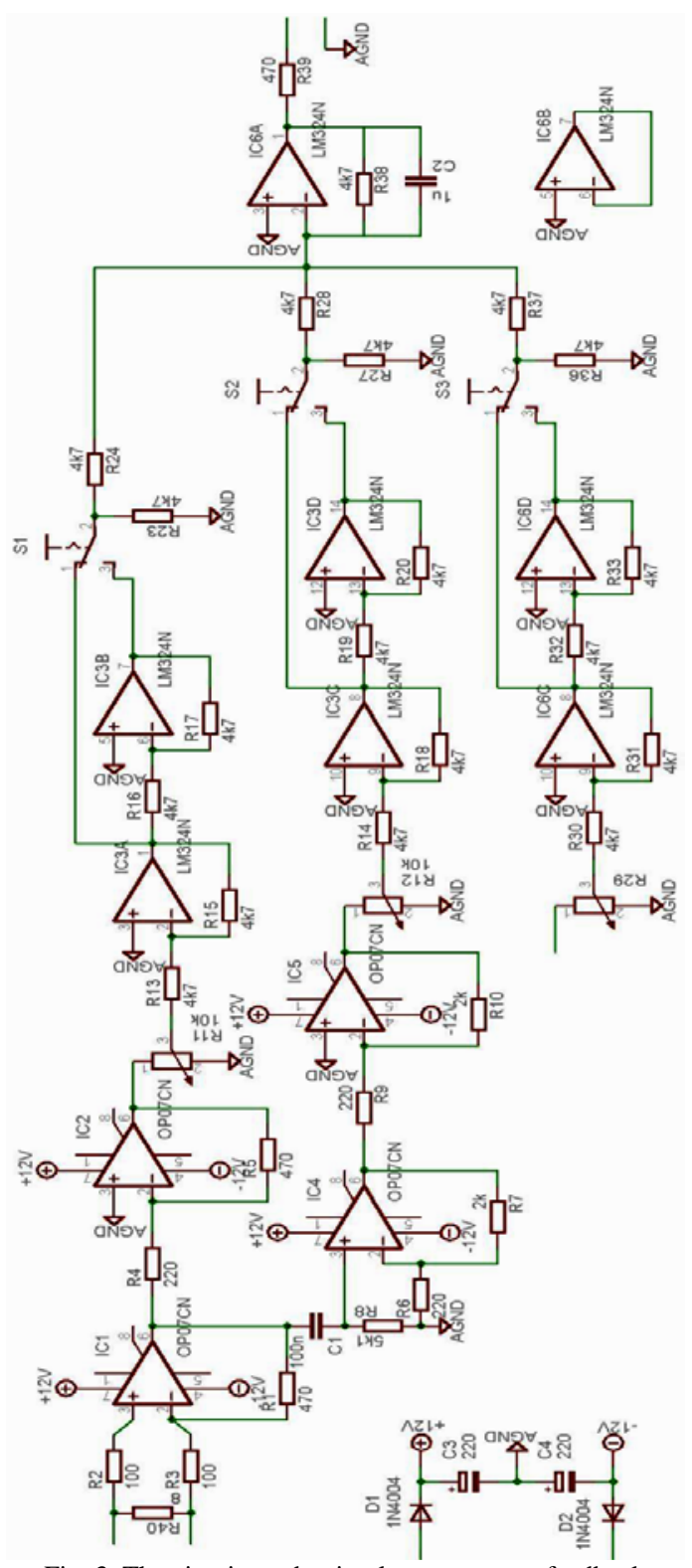

Fig. 3. The circuit used to implement current feedback (proportional/derivative) and acceleration/velocity feedback.

The functioning principle of the speaker allow the experimental study of the control system of the mechanical transmissibility.

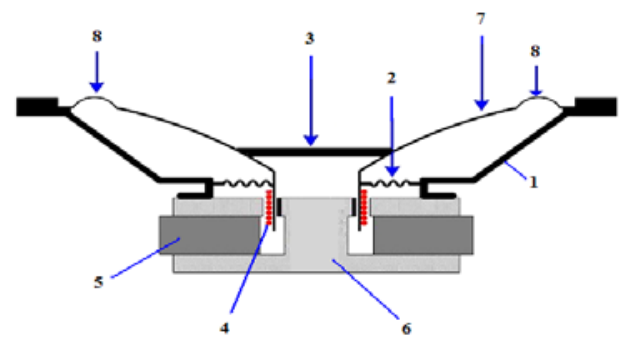

Fig. 4. Constructive elements of the speaker and those which are equivalent to the components of the adjustment system of mechanical mobility. 1-speaker casing, 2-spring, 3- system with mass $\mathrm{m}$ to be isolated, 4-command coil, 5-magnet, 6-metallic armature, 7-conical membrane of the speaker, 8 - embossed area of membrane (equivalent of an elastic element of spring).

The selection of this type of speaker was made because it 
meets together, in one device, all the electromechanical parts of the studied system: the object to be isolated from the action of external forces is represented by the speaker diaphragm, which has mass $\mathrm{m}$, the spring and the damper from the structure of the studied system, with constants $\mathrm{k}$ and $\mathrm{c}$, are equivalent with the elastic damping system that supports the speaker diaphragm and the electrodynamic actuator that generates the force Fem is composed by the coil and his electromagnetic circuit (magnet and metallic armature).

The main technical parameters of speaker, considered as an electrodynamic actuator are presented in Table I.

TABLE I: THE MAIN PARAMETERS OF SPEAKER
\begin{tabular}{|l|l|l|l|}
\hline No. & Parameter & UM & Value \\
\hline 1 & $\mathrm{~L}$ & $\mathrm{H}$ & 0,00056 \\
\hline 2 & $\mathrm{R}$ & $\Omega$ & 8,00 \\
\hline 3 & $\mathrm{~m}$ & $\mathrm{Kg}$ & 0,0434 \\
\hline 4 & $\mathrm{c}$ & $\mathrm{N} /(\mathrm{m} / \mathrm{s})$ & 2,2305 \\
\hline 5 & $\mathrm{k}$ & $\mathrm{N} / \mathrm{m}$ & 2452,5 \\
\hline 6 & $\mathrm{k}_{\mathrm{m}}$ & - & 4 \\
\hline 7 & $\mathrm{k}_{\mathrm{e}}$ & - & 4 \\
\hline
\end{tabular}

To perform experiments for transmissibility of the speaker, was used a stand as the one shown in the Fig. 5.

The stand of carrying out the experiments include:

- the subject studied, in this case the electrodynamic actuator (the electodinamic speaker);

- an impedance analyzer capable of generating sine and sweep sine signals ( 0 to $120 \mathrm{kHz}$ ), which represents the primary element to control the stand and for the registration/processing of the data obtained as a result of the experimental work; the settings are indicated in Table I;

- two piezoelectric accelerometers to measure the acceleration of the base and of part 3 from Fig. 4;

- one electrodynamic vibration exciter with adjustable parameters, which is used to apply to the studied system controlled vibration, necessary for the determination of response to the operating conditions;

- a electrical power source of adjustable voltage and current, required for the application of continuous or variable voltages to supply the circuit form Fig. 3;

tools and clamping devices, for mounting or handling the studied system, which help to set on or positioning the system (speaker) and accessories (transducers, sensors etc.).

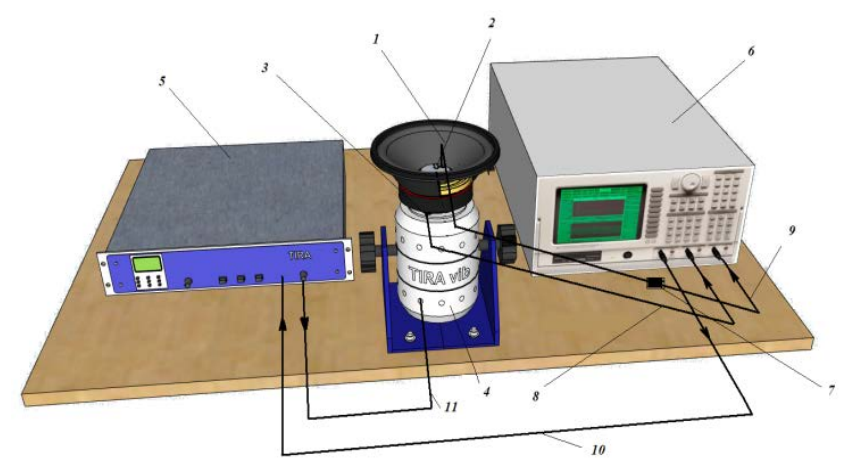

Fig. 5. The schematic diagram of the stand for experimental study of the control system of mechanical mobility. 1-speaker, 2 - accelerometer, 3 force transducer, 4 - electrodynamic vibration exciter, 5 - power amplifier, 6 - signal analyzer, 7 - preamp signal, 8 - input signal power, 9 - input signal .acceleration, 10 - control signal for vibrator parameters, 11 - signal power for vibrator.
The experimental results are indicated in Fig. 6 and confirm the simulated behavior.

TABLE II: IMPEDANCE ANALYZER SETTINGS

\begin{tabular}{|l|l|}
\hline Measurement & Frequency response \\
\hline Measurement type & Swept Sine \\
\hline No. of measuring points & 1000 \\
\hline Starting frequency & $10 \mathrm{~Hz}$ \\
\hline Stop frequency & $110 \mathrm{~Hz}$ \\
\hline Sweep Type (sweep) & Linear \\
\hline Amplitude max & $1000.0 \mathrm{mV}$ \\
\hline Sampling duration & $7.8125 \mathrm{~ms}$ \\
\hline No. of sampling cycles & 5 \\
\hline Integration time & $15.625 \mathrm{~ms}$ \\
\hline No. of integration cycles & 5 \\
\hline Acceleration scale 1 & $1000 \mathrm{~m} / \mathrm{s}^{2} / \mathrm{V}$ \\
\hline Acceleration scale 2 & $1000 \mathrm{~m} / \mathrm{s}^{2} / \mathrm{V}$ \\
\hline
\end{tabular}

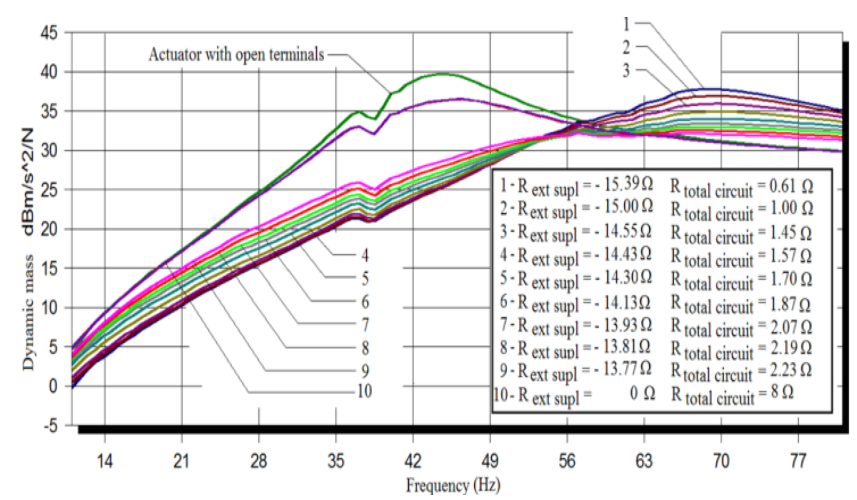

Fig. 6. The variation of the dynamic mass for different values of the electrical resistance, obtained through current feedback.

A very low value of resistance produces the highest attenuation at the mechanical resonance but creates new problems at the electromechanical resonance. An optimum value exists producing the highest attenuation on a large frequency range.

Further developments will focus on modeling practical construction aspects: influence of the fixing layer between the coil and the mobile mass; the vibration modes due to the distributed mass and elasticity of the sustension springs; the energy leakeage through Eddy currents and the effect of magnets non-linear magnetisation curve.

\section{CONCLUSION}

The study proved the possibility to adjust the global electrical parameters using a current loop, both by simulation and experimental and also the procedures to cancel some of them and use the others in order to modify the total equivalent mechanical parameters (mass-spring-viscous). The mobility, impedance and dynamic mass can be controlled much easier than for a pure mechanical system. The ideas of designing the loop were implemented using simple analogue circuits. The solution requests care due to the possibility of having a feedback which can aggravate the system behavior, producing a reduction of the damping and even an oscillating behavior, as observed during the tests.

\section{REFERENCES}

[1] C. M. Harris and A. C. Piersol, HARRIS' Shock and Vibration Handbook, 5th ed. New York, NY: McGraw-Hill, 2005. 
[2] D. J. Mead, Passive Vibration Control, Chichester, New York: Wiley, 1999.

[3] C. R. Fuller and S. Elliot, Active Vibration Control, 1st ed. Academic Press, 1997.

[4] A. Preumont, Vibration Control of Active Structures: An Introduction (Solid Mechanics and Its Applications), 3rd ed. Springer - Verlag Berlin Heidelberg Publisher, 2013.

[5] S. M. Savaresi, L. Dugard, C. Poussot-Vassal, O. Sename, and C. Spelta, Semi-Active Suspension Control Design for Vehicles, 1st ed. Elsevier Science, 2010.

[6] Table Stable. [Online]. Available: http://www.tablestable.com/en/products/list/2/ http://www.moog.com/products/vibration-suppression-control/

[8] R. Banik, Six Degree of Freedom Active Vibration Isolator using Voice Coil Motor: Design and Control, Lambert Academic Publishing, 2010.

[9] Active Vibration Control. [Online]. Available: http://www.lord.com/products-and-solutions/active-vibration-control

[10] D. Abramovitch and G. Franklin, "A brief history of disk drive control," IEEE Control Systems Magazine, vol. 22, no. 3, pp. 28-42, June 2002.

[11] D. Paulitsch, K. I. Okamoto, and K. Utsunomiya, "Self-sensing active damping guide roller for elevators," in Proc. VDI-Berichte 1753, 5.VDI-Mechatronik Tagung 2003, Fulda, Germany, 7-8 May, 2003, pp 91-110.

[12] E. De Boer, "Theory of motional feedback," IRE Trans. on Audio, vol. 2, pp. $15-21,1961$

[13] R. L. Tanner, "Improving loudspeaker response with motional feedback,” Electronics, pp. 142, pp. 228-240, March 1951.

[14] H. W. Holdaway, "Design of velocity-feedback transducer systems for stable low-frequency behavior,” IEEE Trans. on Audio, pp. 155-173, vol. AU-11, no. 5, 1963.

[15] H. W. Holdaway, "Controlling the upper-frequency characteristics of velocity-feedback loudspeaker systems," IEEE Trans. on Audio, pp. 174-182, vol. AU-11, no. 5, 1963.

[16] R. E. Werner and R. M. Carrell, "Application of negative impedance amplifier to loudspeaker systems," Journal of the Audio Engineering Society, vol. 6, no. 4, pp. 240-243, Oct. 1958.

[17] F. E. Terman, Radio Éngineers'Handbook, McGraw Hill, London, 1950.

[18] O. Y. Matsuda and K. Hashitani, "Self-sensing active vibration control using the moving-coil-type actuator," Journal of Vibration and Acoustics, vol. 117, pp. 411-415, October 1995.

[19] M. R. Bai and H. Wu, "Robust control of a sensorless bass-enhanced moving-coil loudspeaker system,” J. Acoust. Soc. Am., vol. 105, no. 6 , pp. 3283-3289, June 1999.

[20] C. Paulitsch, P. Gordonio and S. J. Elliot, Investigation of an Electrodynamic Actuator for Self-Sensing Active Vibration Control, Univ. of Southampton, Institute of Sound and Vibration Technical memorandum 917, August 2003.

[21] M. A. Mitiu and C. D. Comeaga, "Dynamic response control for a mass-spring-viscous damper system by use of an additional electro-mechanical impedance,” UPB Scientific Bulletin, vol. 75, no. 4, pp. 149-162, 2013.

[22] M. A. Mitiu, C. D. Comeaga, O. G. Dontu, and G. I. Gheorghe, “The control of the mechanical impedance of a system with 1DOF by replacing the dynamic absorber with an electro-mechanical system with adjustable parameters," in Proc. MECAHITECH'13 Conference, pp. 229-238, 2013.

[23] A. M. Mitiu and D. Comeagă, "The control of transmissibility and mechanical impedance using electrodynamic actuators," in Proc. the 8th International Symposium on Advanced Topics In Electrical Engineering, May 2013.

[24] A. M. Mitiu, D. Comeagă, and O. Dontu, "The control of vibration transmissibility using an electrodynamic actuator - passive solution," in Proc. International Conference on Smart Systems in all Fields of the Life-Aerospace, Robotics, Mechanical Engineering, Manufacturing Systems, Biomechatronics, Neurorehabilitation and Human
Motricities, ICMERA 2013, October 2013 and Applied Mechanics and Materials, vol. 436, pp. 158-165, 2013.

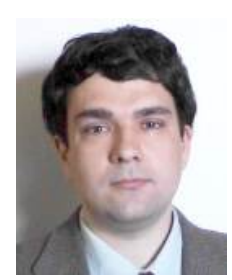

Constantin D. Comeaga was born in Bucharest, Romania, 1971. Constantin D. Comeaga received BSc. in Mechanical Engineering in 1994 and the PhD degree in technical acoustic and mechanical vibrations in 1999 from Politehnica University of Bucharest, Romania. The major fields of study are mechatronics, vibration and noise emission control, smart structures and robotics.

He works as a professor at University Politehnica of Bucharest, Romania, Faculty of Mechanical Engineeringa and Mechatronics, Department of Mechatronics. He is scientific director of Research and Development Center for Mechatronics. Below is the selection of papers: C. D. Comeaga, C. G. Alionte., "The control of a positioning system using bi-axial variable reluctance actuator," in 2010 IEEE International Conference on Automation Quality and Testing Robotics (AQTR), Cluj-Napoca, Romania, 2010, pp. 1-6; C. D. Comeaga, G. Ionascu, E. Manea,A. Sandu, L. Bogatu, D. Besnea, "Influence of technological parameters on the dynamic behavior of a MEMS accelerometer,” 2010 International Semiconductor Conference, Oct. 2010, Sinaia, Romania, pp. 243-246; C. Nitu, B. S. Gramescu, C. D. Comeaga, A. O. Trufasu, "Optomechatronic system for position detection of a mobile mini-robot," IEEE Transactions on Industrial Electronics, vol. 52, issue 4, pp. 969-973, 2005.

His previous research deals with ultrasonic robotics, inch-worm motors, hysterezis and non-linearities compensation in piezoelectric actuators, semi-active control of damping using composite structures with piezoelectric patches. Current research deals with piezoelectric and electromagnetic actuated MEMS, smart sensors and electrodynanic actuators for vibration control.

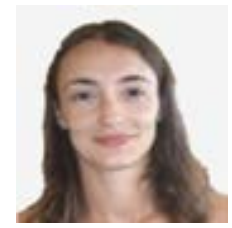

Mihaela Mitiu was born in Bucharest, Romania, 1978. She received her BSc. in mechanical engineering and mechatronics from Politehnica University of Bucharest, Romania, in 2003. She received in 2004 her MSc in advanced mechatronics and the $\mathrm{PhD}$ degree in mechanical engineering from Politehnica University of Bucharest, Romania. Her research interests lie in the area of new mechnic systems for vibration attenuation by active and semi-active control systems.

She works as a senior scientific researcher at The National Institute for Research and Development in Environmental Protection, Bucharest, Romania.

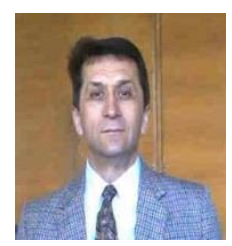

Octavian G. Dontu was born in Ploiesti Romania, 1947. He received the $\mathrm{BSc}$, in precision mechanics (fine mechanics) in 1970 from University Politehnica and obtained the $\mathrm{PhD}$ title in laser engineering systems for material processing in 1985 .

He works as a professor at University Politehnica of Bucharest, Romania, Faculty of Mechanical Engineeringa and Mechatronics, the Department of Mechatronics. He is $\mathrm{PhD}$ supervisor and in the last 10 years taught classes on "Laser Processing Technology" at E.N.S.A.M. Aix en Provence, University Bordeaux-Sciences et Technologies, France and University West Macedonia Tei Kozani, Greece.

In his scientific career has published over 130 scientific papers in mechatronics field and laser processing technologies, being member of Romanian Mechatronics Society, International Mechatronics Society and International Laser Club. 